\title{
SOCIOLOGIA GIURIDICA E STORICA, STORIA DEL DIRITTO E, IN BRASILE, “ANTROPOFAGIA GIURIDICA"
}

\section{LEGAL AND HISTORICAL SOCIOLOGY, LEGAL HISTORY, AND, IN BRAZIL, “JURIDICAL ANTHROPOPHAGY”}

Mario G. Losano*

\begin{abstract}
RIASSUNTO: Attraverso l'analisi del libro História do direito pelos movimentos sociais, di Gustavo Silveira Siqueira, questo articulo studia, alla luce della teoria del diritto, i problemi e le possibilità della così chimata "antropofagia giuridica", l'approccio metodologico da Siqueira proposto per la storia del diritto in Brasile.

PAROLE CHIAVE: “Antropofagia giuridica”. Metodologia. Storia del diritto in Brasile. Teoria del diritto.
\end{abstract}

ABSTRACT: Through the analysis of the book História do direito pelos movimentos sociais, by Gustavo Silveira Siqueira, this article studies, in the prism of legal theory, the problems and possibilities of the so-called "juridical anthropophagy", the methodological approach proposed by Siqueira for Brazilian legal history.

KEYWORDS: Brazilian legal history. “Juridical Anthropophagy”. Legal theory. Methodology.

Indice: 1 Discipline dai confini incerti. - 2 Breve storia della storia del diritto in Brasile. -3 L'“antropofagia” nella cultura brasiliana. - 4 Il libro di Gustavo Siqueira. - 5 L'analisi storicogiuridica dello sciopero ferroviario del 1906.

\section{DISCIPLINE DAI CONFINI INCERTI}

Nel fiorire di studi sulla storia del diritto in Brasile, un recente volume analizza la percezione del diritto di sciopero all’inizio del Novecento nei movimenti sociali e nei magistrati coinvolti. Rispetto alle storie giuridiche tradizionali che si fondano soprattutto sul diritto positivo, questa ricerca tenta una metodologia nuova per costruire una "storia del diritto prodotto dai movimenti sociali”: dichiarazione di principio che dà il titolo al volume stesso (SIQUEIRA, 2014). ${ }^{1}$

\footnotetext{
* Dottore honoris causa presso l'Università di Hannover (Germania); della Repubblica, Montevideo (Uruguay); Carlos III, Madrid (Spagna); Professore onorario presso l'Università Federale di Pernambuco (Brasile). Professore Emerito di Filosofia del Diritto e Introduzione alla Informatica Giuridica presso la Facoltà di Giurisprudenza dell'Università del Piemonte Orientale, Alessandria (Italia). Professore presso la Scuola di Dottorato dell'Università degli Studi di Torino (Italia).E-mail: mario_losano@yahoo.it

${ }^{1}$ D'accordo con Siqueira, ho tradotto "História do direito pelos movimentos sociais" con "storia del diritto prodotto dai movimenti sociali”, per rendere chiaro che il libro ha per oggetto il diritto prodotto dai movimenti sociali, e non la
} 
Il caso concreto su cui saggiare questa storia-non-formalistica del diritto - o si tratta d'una storia del diritto-non-formalistico? - è lo sciopero ferroviario svoltosi nel 1906 nello Stato di São Paulo. Il suo svolgimento e le polemiche giuridiche che lo accompagnarono sono descritte nell'ultimo capitolo del libro, mentre i tre capitoli precedenti approfondiscono gli aspetti metodologici della ricerca. Sul contenuto dell’intero volume ritornerà in dettaglio il $\S 4$, mentre il $\S$ 5 analizza i problemi metodologici insiti nella scelta dell'Autore. Vanno però illustrati preliminarmente alcuni aspetti culturali - tipicamente brasiliani - che stanno alla base di questo libro. Anzitutto il $\S 2$ colloca questa proposta metodologica nel contesto della storia giuridica del Brasile, la cui evoluzione è radicalmente diversa non solo da quella europea, ma anche da quella degli altri paesi dell’America Latina. Il $\S 3$ traccia brevemente i contorni dell’idea ispiratrice di questa proposta, cioè dell'“antropofagia giuridica”, concetto che al lettore europeo può apparire bizzarro, ma che ha precise e profonde radici nella cultura brasiliana moderna: per questo il $\S 3$ tratta del movimento modernista e della "Semana de Arte Moderna” di São Paulo, nel 1922. A queste radici si richiama Siqueira: nel 1928, "Oswald de Andrade diede nel Manifesto antropofágico una connotazione politica e ideologica dell'antropofagia” nel tentativo "di costruire una tradizione nazionale che potesse dialogare con le avanguardie europee” (p. 97), in particolare con il Futurismo italiano che fu una delle fonti del modernismo brasiliano. Il nome di Oswald de Andrade verrà citato per esteso nelle pagine che seguono, perché nello stesso ambiente e nello stesso movimento operava Mário de Andrade, che di Oswald era non parente, ma amico, almeno fino alla rottura dei loro rapporti. Così, dopo essere stati il Castore e Polluce ovvero l'Oreste e Pilade del modernismo brasiliano, ne divennero il Castore e Pilade ovvero l'Oreste e Polluce: eroi abbinati ma disomogenei.

L’opera di Siqueira ripropone in una formulazione originale il problema dei confini tra storia contemporanea del diritto, sociologia del diritto e sociologia storica del diritto ${ }^{2}$ : problema insoluto perché probabilmente insolubile.

\section{BREVE STORIA DELLA STORIA DEL DIRITTO IN BRASILE}

In Brasile la storia del diritto come disciplina universitaria è recentissima - a differenza di quanto è avvenuto in altri Stati dell’America Latina, soprattutto in Messico, Argentina e Cile -

storia del diritto vista da quei movimenti. Nel mio testo, l’indicazione fra parentesi di una pagina si riferisce al volume di Siqueira. I nomi vengono scritti nella grafia attuale, che non sempre corrisponde a quella di oltre un secolo fa.

${ }^{2}$ Ritorno così ai problemi affrontati nel volume da me curato. Cf. LOSANO, Mario G. Storia contemporanea del diritto e sociologia storica. Milano: Franco Angeli, 1997. 
poiché si sviluppa a partire dal 1990 e trova il suo fondamento normativo in un decreto ministeriale del 1994 (Portaria/MEC 1886 del 1994).

Questa peculiarità va ricercata nella storia generale del Brasile ed è stata illustrata in un dettagliato articolo di Ricardo Fonseca (2012), attuale presidente dell'Instituto Brasileiro de História do Direito (IBHD) ${ }^{3}$. Vi si ricorda come le università brasiliane vennero fondate soltanto a partire dall’indipendenza nel 1822, mente prima di esse la formazione dei giuristi avveniva in Portogallo e soprattutto a Coimbra. "A Olinda ed a São Paulo [le prime due facoltà di diritto in Brasile] si realizza un curriculum di studi senza la storia del diritto ed anche senza il diritto romano. Sin dall'inizio è chiaro l'indirizzo pratico, volto a formare burocrati, e non intellettuali, [...] per fornire personale specializzato al giovane Stato da poco indipendente” (cpv. 8).

In realtà, alcuni decreti tentarono di introdurre l’insegnamento della storia del diritto già tra il 1885 e il 1891, ma la disciplina venne soppressa nel 1901: dopo questi 15 anni la storia del diritto scomparve dalle facoltà giuridiche brasiliane, anche se non mancarono studi storico-giuridici dovuti però soltanto all’interesse di singoli studiosi, quali Isidoro Martins Jr. (1895), autore di una storia giuridica nel 1895 o, nel corso del secolo XX, Miguel Reale, Nelson Saldanha o Machado Neto. Martins riflette l'atmosfera evoluzionistica dominante a Recife (che ebbe la sua figura più rilevante in Tobias Barreto ${ }^{4}$ ), mentre i restanti provenivano dalla filosofia del diritto e quindi praticavano la ricerca storica "nell'ambito e secondo le preoccupazioni d'una filosofia del diritto attenta alla temporalità, soprattutto alla temporalità delle idee giuridiche. Nel Brasile non si faceva dunque della storia del diritto come avveniva nello stesso periodo in Europa e in molti luoghi dell’America Latina” (cpv. 13).

Solo con il già ricordato decreto del 1994 il curriculum delle facoltà giuridiche si aprì ufficialmente allo studio delle materie storiche. Con il XXI secolo questa apertura si accompagnò all'incremento delle facoltà giuridiche, soprattutto private, che oggi in Brasile sono circa 1100: secondo l'immagine di Fonseca, dopo decenni di “deserto” esistevano finalmente le premesse per l’esplosione "vulcanica” delle cattedre di storia del diritto.

Fonseca propone due possibili spiegazioni per questo singolare percorso. La prima segue le orme di António Hespanha (1978) (cpv. 16), che spiega l'affermarsi della storia del diritto in Portogallo nel secolo XIX come strumento per spiegare l'affermarsi delle caute riforme liberali e

\footnotetext{
${ }^{3}$ L'immagine del titolo si riferiscono all'andamento della storia giuridica in Brasile, che l'autore vede come un deserto sino alla fine del XX secolo, sostituito oggi da un'esplosione di scritti su questo tema. D'ora in poi citato così: Fonseca, $\mathrm{O}$ deserto.

${ }^{4}$ Su questo giurista che introdusse Rudolf von Jhering in Brasile, cf. LOSANO, Mario G. Un giurista tropicale. Tobias Barreto fra Brasile reale e Germania ideale. Roma: Laterza, 2000.
} 
segnare quindi l'abbandono dell'assolutismo. In realtà il Brasile non conobbe la rottura istituzionale che caratterizzò gli Stati ispanofoni dell’America Latina: infatti l'occupazione napoleonica troncò i rapporti delle colonie spagnole con la madrepatria e provocò le dichiarazioni d’indipendenza accompagnate dalle lotte tra gli indipendentisti, da una parte, e i lealisti e le truppe spagnole dall'altro. L’invasione napoleonica obbligò la corte del Portogallo ad imbarcarsi per il Brasile, che passò così da colonia a Regno e poi a Impero senza spargimento di sangue. Quindi, "in questo periodo, la storia del diritto in Brasile non esercitò alcuna funzione”: la storia istituzionale del Brasile manifesta una sostanziale continuità tanto nel momento dell'indipendenza quanto nella successiva proclamazione della repubblica, cosicché non si avvertiva "la necessità di stabilire nuove forme di legittimazione” (cpv. 17).

La seconda spiegazione fornita da Fonseca si riferisce alla peculiarità del diritto romano così com’era insegnato nel Brasile dal 1854 fino al codice civile del 1916: era un "diritto romano vigente” di stampo tedesco e serviva non tanto a "storicizzare la ricchissima esperienza romana”, quanto a fungere da "introduzione allo studio della dogmatica del diritto privato" (cpv. 20). Anche il romanista tedesco Gustav Hänel aveva ritenuto utile richiamare l'attenzione sulla permanenza in Portogallo del diritto romano sino alle soglie del XIX secolo (HÄNEL, 1835) ${ }^{5}$.

“Come il diritto romano sembra aver bloccato per molto tempo la 'rinascita' della storia del diritto, così il sorgere della storia del diritto sembra aver avuto un impatto sull'ambito accademico del diritto romano”, anche perché quest'ultimo viene sempre meno insegnato, tanto che oggi in molte nuove università non è più presente (cpv. 19, nota 11). Inoltre, dove lo si studiava, il diritto romano esauriva la spiegazione storica anche dei codici moderni, mentre era del tutto trascurata la storia del diritto medievale e moderno. Solo col XXI secolo "si supera questo deserto" e la disciplina si presenta oggi come "un vulcano in eruzione continua”, come un'esplosione che è “di metodo, di temi, di stili e di tendenze” (cpv. 22).

Nelle attuali correnti della storia del diritto brasiliana, Fonseca distingue due contrapposizioni: anzitutto quella tra una visione dogmatica ed una critica del diritto, e poi quella tra una visione sintetica ed una analitica di questo stesso oggetto.

Nella prima contrapposizione, al diritto come "il risultato inevitabile di tutto un processo storico” si oppone una visione “critica” del diritto e del suo divenire. A quest'ultima corrente va la simpatia di Fonseca e di molti autori brasiliani contemporanei. La si può far risalire alla "forte influenza critica di alcuni autori stranieri [...] come Michael Stolleis, Pietro Costa, Paolo Cappellini, Carlos Petit ecc. Tuttavia, all’interno di questo approccio, credo che emergano, -

\footnotetext{
${ }^{5}$ Che si richiama alle “Memorias de Literatura Portugueza” dell’ Accademia Reale delle Scienze di Lisbona.
} 
continua Fonseca, - due storici europei del diritto: Paolo Grossi e António Manuel Hespanha. Il primo, già tradotto in modo rilevante in Brasile, è portatore di un forte messaggio sulla funzione critica, relativizzante e demistificatrice della missione dello storico del diritto”, mentre il secondo “richiama l'attenzione sulla funzione nociva che la storia del diritto, mal informata dal punto di vista metodologico, può assolvere”, limitandosi “alla pura storia delle leggi” o “delle grandi scuole” e trascurando "i contesti complessi" e "le rotture e le discontinuità che caratterizzano il passato giuridico" (cpv. 29).

La seconda contrapposizione viene ricondotta al consolidarsi d’una disciplina nel contesto universitario brasiliano che conta più di mille facoltà di diritto: questo vasto mercato deve essere approvvigionato con opportuni prodotti editoriali adatti a un pubblico studentesco, cioè alle prime armi con il diritto. Per questa ragione commerciale "i libri in questione tendono al maggior schematismo possibile” (cpv. 30). Fonseca però non è contro i manuali, bensì contro i cattivi manuali, quelli cioè di livello elementare ed orientati al consumo, perché tentano una sintesi quando ancora in Brasile non v’è "sufficiente materiale d'analisi", che consiste in "articoli scientifici, indagini universitarie, relazioni a congressi e altri materiali monografici” (cpv. 33). Per questi ultimi prodotti culturali si è aperto "un futuro promettente per la storia del diritto in Brasile” (cpv. 34): futuro che si presenta caratterizzato da una forte interdisciplinarità. "Sono frequenti le analisi storico-giuridiche che uniscono un'analisi delle fonti (tanto archivistiche quanto dottrinali) con forme di analisi fortemente influenzate da sociologi, politologi e filosofi (come Max Weber, Karl Marx e alcune sue derivazioni latino-americane, Pierre Bourdieu, Michel Foucault ecc.)”: queste forme di analisi “generalizzante” rafforzano il profilo “critico” di queste ricerche (cpv. 37).

In conclusione, una concezione critica della storia del diritto come quella proposta da Fonseca, porta "la disciplina a svolgere una funzione di 'straniamento' rispetto al passato (forse anche per le diffuse conoscenze del sapere antropologico), a una relativizzazione dei percorsi nel tempo. [...] La conoscenza storico-giuridica svolge spesso la funzione di criticare e di dedogmatizzare le opzioni del diritto attuale, mostrandone la contingenza e precarietà. La storia del diritto si presenta così chiaramente come una disciplina critica” (cpv. 30).

Il richiamo alle "conoscenze del sapere antropologico” apre così le porte all’esame del libro di Gustavo Siqueira e all'uso che vi si fa della nozione di “antropofagia”. Siqueira può dare per scontato che il lettore brasiliano conosca il contesto culturale in cui prese forma il movimento “antropofagico” e può quindi calare nella storia del diritto un termine e una concezione che, nella cultura brasiliana, circolano in un’area molto più vasta. Invece per il lettore non brasiliano, prima di 
passare all'analisi del suo libro, è opportuno soffermarsi sul concetto di “antropofagia” ricorrente in quel libro.

\section{L’“ANTROPOFAGIA” NELLA CULTURA BRASILIANA}

La denominazione del movimento brasiliano d'avanguardia si ricollega direttamente al manifesto di Oswald de Andrade (1890-1954) pubblicato nel 1928 con il titolo Manifesto Antropófago. Con questo richiamo conturbante e inconsueto egli voleva riportare artisti plastici e scrittori alle radici autoctone dell’America latina, abbandonando le imitazioni straniere.

Infatti proprio dai Caraibi i navigatori europei avevano portato in Europa le prime notizie dei popoli “cannibali” incontrati: già Cristoforo Colombo, nel suo Diario del primer viaje, aveva registrato la crescente agitazione della ventina di indios che aveva a bordo quando la "Santa María” si avvicinava sempre più all’isola di Haiti, “a quien aquellos Indios llamaban Bohío”, perché lì viveva gente "que se llamaban 'Canibales"”, di cui gli altri indios avevano grande paura "porque los comían”. Questa è dunque l'origine della parola “caníbal”, che dallo spagnolo è passata alle altre lingue intrecciandosi con la parola greca 'anthropòphagos' in una complessa storia etimologica che altri ha già ricostruito (LEBEK, 2001).

Il fatto che "la fantasia dell’Europa occidentale sia affascinata da fenomeni culturali nonoccidentali come il cannibalismo" è così spiegato da etnologi e antropologici: "I cannibali sono l'altro, l'estraneo a un livello culturale e sociale più basso, da cui può venire una minaccia [...]. Il delitto considerato disumano giustifica la persecuzione, la sottomissione, lo sfruttamento e l’annichilamento di queste razze primitive da parte del conquistatore civilizzato”6.

Contro questo delitto Carlo V ordinava nel 1523: "Mandamos que se defienda, notifique y admoneste a todos los naturales de nuestra Indias, que no tengan ydolos donde sacrifiquen creaturas humanas ni coman carne humana ni hagan otra abominaciones contra nuesta santa fe cathólica”7 . I resoconti spesso fantasiosi diedero origine tra l'altro anche a un'iconografia tra l'ingenuo e il raccapricciante che durerà per secoli e che comunque marchierà in Europa l'immagine del Tropico sudamericano, dai Caraibi al Nord-Este brasiliano ${ }^{8}$.

\footnotetext{
${ }^{6}$ La citazione è tratta da Roswitha Burwick, “Wenn er fett ist, so will ich ihn essen” (LEBEK, cit., p. 242), che analizza i riferimenti antropofagici nelle fiabe romantiche di fratelli Grimm; il volume registra numerosi altri esempi tratti dalla letteratura europea o angloamericana, ma non menziona il fenomeno sudamericano, per il quale si rinvia a JÁUREGUI, infra, nota 8.

${ }^{7}$ Carlos V, Recopilación 76, 23 de julio de 1523.

${ }^{8}$ Carlos A. Jáuregui, della Vandelbilt University, ha pubblicato un pregevole studio che copre tutta l'America meridionale, con una vasta iconografia (35 tavole) e bibliografia (p. 605-54). Si veda in particolare il Cap. V,
} 
Oswald de Andrade si riappropria di questo mito per ricavarne una teoria culturale che si colloca nel contesto del Modernismo brasiliano e che cerca una risposta al quesito comune alle avanguardie latino-americane dell'inizio del XX secolo: “Come far parte del mondo senza dissolversi, senza fondersi nel globale?”, cioè “come essere brasiliani senza rinunciare ai beni culturali della modernità, che includono, per esempio, gli strumenti tecnici delle euro-avanguardie futurismo, cubismo, Dadà, surrealismo - e la cinematografia, la psicoanalisi ecc.?” (JÁUREGUI, 2008, p. 425).

La ricettività del pubblico colto per la metafora antropofagica era legata anche alle scoperte antropologiche del XIX secolo, che avevano suscitato un dibattito ancora in corso nel XX secolo sul cannibalismo (alimentare o funerario) dell'uomo preistorico. A Parigi, nel 1920 Francis Picabia e altri dadaisti danno pubblica lettura del "Manifeste Cannibale Dada" e pubblicano la rivista “Cannibale”, “avec la collaboration avec tous les dadaïstes du monde”. Nel 1913 in Totem e tabù Freud spiega come l'individuo tenda a divorare l'oggetto del suo desiderio: e Oswald de Andrade cita Freud varie volte nel suo Manifesto antropofágico. "Questo dibattito di lunga durata non poteva che contribuire alla diffusione dell’ideologia antropofagica al di là delle frontiere del paese”, cioè del Brasile (SILVA, 2009, P. 48).

L’inizio del movimento modernista in Brasile può essere collocato intorno al 1916-1922 e il suo completamento nel 1945: molte esitazioni circondano la data d'inizio, poche invece la data finale $^{9}$. Il richiamo al futurismo italiano e alle altre avanguardie europee è ambivalente: da un lato il Modernismo ne recepisce l'impatto antitradizionalista, ma dall'altro se ne distacca per seguire una via autoctona, che liberi il Brasile dalla coazione a ripetere ciò che viene dall’Europa. Menotti del Picchia, esponente di rilievo del Modernismo, esprime chiaramente questa ambivalenza rifiutando anzitutto il termine "futurismo" per il movimento brasiliano, perché ciò significa "usare, con improprietà, un termine che in Europa è servito a designare la reazione geniale ed idiota di un'orda di avanguardisti reazionari, i cui generali erano talenti e i cui aderenti erano imbecilli”; in Brasile, il modernismo "non accetta i principi dogmatici della scuola di Marinetti, anche perché il futurismo di São Paulo odia tutto ciò che è scuola"10.

Il punto culminante del Modernismo fu la "Settimana d’Arte Moderna”, organizzata a São Paulo dall'11 al 18 febbraio 1922 come contro-celebrazione rispetto alle manifestazioni ufficiali che

Antropofagía: consumo cultural, modernidad y utopía, p. 393-460. Cf. JÁUREGUI, Carlos A. Canibalia: canibalismo, calibanismo y antropología cultural en América Latina. Madrid: Iberoamericana, 2008.

${ }^{9}$ Luciana Stegagno Picchio, nella sua Storia della letteratura brasiliana (PICCHIO, Luciana Stegagno. Storia della letteratura brasiliana. Firenze: Sansoni, 1972), colloca Oswald de Andrade e il terrorismo culturale (p. 484-8) nel contesto del Modernismo brasiliano (p. 461-520).

${ }^{10}$ Menotti del Picchia, Hélios, 1921, cit. in PICCHIO, cit., p. 470. 
si svolgevano in quell'anno per commemorare il centenario dell'indipendenza del Brasile. Il Modernismo così contro-ufficializzato si estese a tutto il Brasile e a tutte le arti. Non aveva un indirizzo politico - rifiutava Marinetti anche a causa dell'abbinamento tra futurismo e fascismo: quest’ultimo aveva preso il potere in Italia proprio nel 1922 - ma raccoglieva qualsiasi forma di protesta contro ogni tradizionalismo.

L’etichetta modernista copre tutto, - sintetizza Stegagno Picchio, - dal momento che il denominatore comune è solo la "libertà". Libertà dal passato "portoghese": dove il nazionalismo autonomista può sfociare tanto nella presa di coscienza creatrice di nuove forme espressive quanto nell'anarchia grammaticale. Dal passato "europeo” in genere: dove il ricupero nativista può stimolare la più feconda e ironica anarchia antropofagica o giungere al più cupo fascismo integralista. Nel Modernismo c’è tutto (PICCHIO, p. 476). ${ }^{11}$

Oswald de Andrade, figura-chiave del Modernismo brasiliano, entra in contatto con le avanguardie europee nel suo soggiorno a Parigi nel 1912, lancia un primo manifesto poetico nel 1924 (Manifesto da Poesia Pau Brasil), e ne radicalizza le idee nel successivo Manifesto Antropófago (o Manifesto Antropofágico) pubblicato nel 1928 nella "Revista de Antropofagia”," che riproduce nella copertina una classica stampa seicentesca con cannibali. Con un linguaggio che richiama quello delle avanguardie da Marinetti a Bréton, Oswald de Andrade propone la “deglutição” metaforica della cultura importata dall’Europa e dall’America del Nord e di quella autoctona dei diversi in Brasile (indios e immigrati d'ogni origine), intendendo con "deglutição” non l'imitarle o il respingerle, ma il farle proprie, creando un'estetica nazionale del Brasile, dalla quale nasca una "poesia d'esportazione”, dopo tanta poesia importata.

Il Manifesto Antropofágico espone in forma provocatoria la reazione di Oswald de Andrade alle tradizioni artistiche e culturali, con una densità di riferimenti e con una concisione di linguaggio che esigono un commento per quasi ogni parola, a partire dal riferimento nel titolo all'antropofagia, concetto illustrato poco sopra, fino alla criptica data del manifesto stesso, dato “A Piratininga” (l’area di São Paulo, in una lingua india) nell’“Anno 374 della deglutizione del

\footnotetext{
${ }^{11}$ L' “Integralismo” fu il fascismo brasiliano. Cf. TRINDADE, Hélgio. Integralismo: o fascismo brasileiro na década de 30. São Paulo: Difel, 1979.

${ }^{12}$ Il titolo originale è Manifesto Antropófago, come si legge nelle due pagine della "Revista de Antropofagia” del 1928 [Anno I, n. 1, p. 3 e 7: riprodotte tra le foto fuori testo dopo la p. 160 in FONSECA, Maria Augusta. Oswald de Andrade (1890-1954): biografia. São Paulo (Secretaria de Estado da Cultura): Art Editora, 1990; il catalogo (a p. 15 riproduce soltanto la p. 3 dell'originale) della mostra realizzata nel 1990, centenario della nascita di Oswald (18901954); ANDRADE, Oswald de. Memorie sentimentali di Giovanni Miramare. Tradução Giovanni Cutolo. Prefácio Giuseppe Ungaretti. Milano: Feltrinelli, 1970]. La forma Manifesto Antropofágico è grammaticalmente più corretta, ma non è conforme all'originale. Il testo integrale del manifesto in portoghese è in http://www.ufrgs.br/cdrom/oandrade/oandrade.pdf, mentre la traduzione in spagnolo è in http://www.ccgsm.gob.ar/areas/educacion/cepa/manifiesto_antropofago.pdf (traduzione ricca delle note esplicative, indispensabili alla comprensione del testo). Una traduzione in francese è in SILVA, cit., p. 157-161.
} 
Vescovo Sardinha”: Oswald de Andrade fa iniziare l'èra brasiliana con il primo atto antropofagico ufficialmente noto, poiché nel 1556 quel vescovo naufragò nel Nordeste e venne divorato dagli indios caetés: non nomen sed omen, visto che 'sardinha' è proprio la Sardina pilchardus della gastronomia portoghese.

Gli enunciati del Manifesto Antropofágico sono lapidari. Eccone l’inizio: “Solo l'antropologia ci unisce. Socialmente. Economicamente. Filosoficamente. | Unica legge al mondo. Espressione mascherata di tutti gli individualismi, di tutti i collettivismi. Di tutte le religioni. Di tutti i trattati di pace. | Tupy or not tupy, that is the question" ${ }^{13}$. Quest'ultima espressione fonde Shakespeare con gli indios "tupí” (etnia numerosa e cannibale) e sintetizza il dilemma brasiliano: tornare alle radici o non tornarci significa esistere o non esistere. Quest'espressione coniata da Oswald de Andrade è ormai entrata anche nel linguaggio corrente ed esprime così a fondo il dilemma brasiliano, che la si ritrova un po’ dovunque. La stessa Luciana Stegnano Picchio l’ha collocata come motto dell’intera sua Storia della letteratura brasiliana.

Ecco poi il richiamo a Freud: “Avevamo la giustizia, codificazione della vendetta. La scienza, codificazione della magia. Antropofagia. La trasformazione permanente del tabù in totem”. Ecco il ritorno alle origini: “Avevamo già il comunismo. Avevamo già la lingua surrealista. L'età dell’oro”. Ecco infine l'unico accenno al diritto, ironizzando sul linguaggio dei giuristi: "Chiesi a un uomo che cos'era il diritto. Mi rispose che era la garanzia dell'esercizio della possibilità. Quest'uomo si chiamava Galli Mathias. Me lo mangiai”14. Costruire un'estetica, un’opera d'arte o letteraria, o una storia del diritto partendo da questo testo è un’impresa pressoché disperata.

Giuseppe Ungaretti, presentando la traduzione d'un libro di poesie di Oswald de Andrade del 1924, coglie l'essenza del suo argomentare: "Per Osvaldo il selvaggio significa un po' sempliciottamente un modo ante litteram di ciò che oggi [nel 1970] si usa chiamare, avendo di rado l'arte di argomentare paradossale e la poesia mordicchiante e allegra di Osvaldo, ‘contestazione’”(ANDRADE, 1970, prefazione). O “provocazione”, quarant'anni dopo: ma queste pagine non possono seguire ulteriormente il fenomeno antropofagico nelle sue varie ramificazioni, perché devono ora concentrarsi sulla sua possibile applicazione alla storia del diritto, proposta nel volume di Siqueira e commentata nel § 5 .

Il Modernismo brasiliano dalle molte anime era destinato a scindersi, generando movimenti nazionalisti come Verde-Giallo, come Anta (Tapiro), o come il regionalismo annunciato nel 1926 dal manifesto di Gilberto Freyre. Il densissimo 1922 fu l'anno della "Settimana d’Arte

\footnotetext{
${ }^{13}$ La traduzione di alcuni passi del Manifesto antropófago (tra cui quelli citati) sono in PICCHIO, cit., p. 482 et seq.

14 Traduzioni mie, tratte dal testo portoghese citato supra, nota 12.
} 
Moderna”, ma anche l'anno della rivolta “tenentista” del Forte di Copacabana, dove alcuni giovani ufficiali morirono per gli ideali di destra che ispireranno l’Integralismo e la dittatura di Getúlio Vargas dal 1930. Il fondatore dell’Integralismo è Plínio Salgado, un autore legato alla "Revista de Antropofagia” che dall'Italia scriveva: "Ho studiato molto il fascismo. Non è esattamente il regime di cui abbiamo bisogno qui [in Brasile], ma è qualcosa di simile” (TRINDADE, 1979, p. 83). L’organo letterario di questa parte del movimento fu la rivista "Verde Amarelo", verde e giallo: i colori della bandiera brasiliana. Essa si contrapponeva a "Pau Brasil”"15 di Osvald de Andrade, che aderì invece alla sinistra politica, iscrivendosi al Partito Comunista Brasiliano e seguendo una traiettoria che fa concludere alla Stegagno-Picchio: “Oswald de Andrade si accorgerà di avere importato dall'Europa il manifesto sbagliato: quello di Marinetti invece di quello di Carlo Marx” (PICCHIO, 1972, p. 476).

In conclusione, il Manifesto Antropófago propone un arsenale di metafore fondate sulla deglutizione e sull'assimilazione dell'altro, che alludono a un'estetica o a un metodo, senza però svilupparli. Come in quasi tutti i testi d'avanguardia, è difficile individuare al suo interno il confine tra la dissacrazione costruttiva e l'elemento ludico o ironico che si esaurisce nella pirotecnia verbale. Qui sta la difficoltà nell'applicare l'antropofagismo culturale a un progetto creativo: come catturare con questo strumento i concetti della storia del diritto? Disporre della rete non vuole ancora dire prendere i pesci, specie se la rete ha le maglie molto larghe.

L’autrice franco-brasiliana Joseane Lucia Silva esamina la presenza dell’antropofagismo di Owald de Andrade nelle arti plastiche, giungendo alla conclusione che esso era uno dei "vettori intellettuali" del Modernismo, influenzato dai movimenti europei con "una forte componente ideologica come il futurismo italiano, il dadaismo e il surrealismo", ma che esso "non ebbe pressoché alcuna conseguenza estetica diretta” perché “i teorici dell’antropofagismo non avevano fissato delle regole che avrebbero permesso agli artisti plastici di raggiungere il loro scopo" (SILVA, 2009, p. 51) ${ }^{16}$. Questa mi sembra l'opinione-chiave da tener presente anche nel tentativo di costruire un'“antropofagia giuridica”.

Quest'ultima incontra un'ulteriore difficoltà - questa volta di tipo ideologico nell'applicazione che Siqueira intende farne al “diritto prodotto dai movimenti sociali”. I modernisti rifiutavano il futurismo, ma poi ne accettavano la magnificazione della modernità tecnologica con frequenti richiami lessicali alla velocità, all'aeroplano, all'automobile o all'elettricità, cioè ai

\footnotetext{
15 “Pau brasil” è l' “albero color della brace” da cui deriva il nome "Brasile”, dato alla sua terra d’origine. Nel CinqueSeicento quel legno veniva esportato in Europa per l'industria tintoria.

${ }^{16}$ Secondo Silva, “[...] l’ideologia antropofagica non fu messa in pratica da alcun artista plastico” salvo la pittrice Tarsila do Amaral, cui dedica la seconda metà del volume.
} 
simboli del trionfo economico nell'entre-deux-guerres. Di quella prosperità i modernisti brasiliani godevano direttamente, come il proprietario agrario Oswald de Andrade, o indirettamente, attraverso l'appoggio economico dato dai "baroni del caffè” alle avanguardie e alla stessa Settimana d'Arte Moderna (finanziata da Paulo Prado ${ }^{17}$ ). Questo condizionamento fa sì che il Modernismo ignori le pesanti ricadute sociali di quello sviluppo economico destinato a dissolversi con la crisi del 1929.

La summa dell'antropofagia culturale di Carlos Jáuregui sottolinea che "questa modernizzazione aveva significati sociali diversi” rispetto al tumulto estetico dei modernisti e che il disagio si manifestava negli “scioperi che, nelle principali città del paese, si succedettero dal 1917 al 1920, nei quali i lavoratori chiedevano aumenti salariali e un miglioramento delle condizioni di vita”. Questi movimenti sociali non interessavano i modernisti e anche la partecipazione di Oswald de Andrade al partito comunista fu di breve durata. "Questi movimenti furono repressi con violenza, - conclude Carlos Jáuregui, - e alcuni dei loro leaders, che erano immigranti, furono espulsi dal paese. Così, mentre da un lato si importavano dall'Europa i telefoni, i quadri di Picasso e le auto sportive, dall’altro si rimpatriavano i sindacalisti” (JÁUREGUI, 2008, p. 400).

Una repressione non minore colpì già nel 1906 lo sciopero ferroviario di cui si occupa Siqueira: ma non sorprende che l'antropofagismo culturale, orientato secondo i condizionamenti fin qui esaminati, fornisca ben pochi strumenti per una storia del diritto che guarda con simpatia ai movimenti sociali oggetto della repressione e vede in essi dei potenziali creatori di un diritto nuovo. Più che strumenti di analisi, fornisce un indirizzo, un invito a guardare la realtà brasiliana e non quella europea o nord-americana.

In conclusione, l'antropofagismo culturale brasiliano è una contestazione o una provocazione che assume una forma artistica lato sensu, ma non offre un insieme di regole da seguire nella sua applicazione a un oggetto culturale. Senza di esse, diviene difficile costruire uno specifico oggetto artistico o scientifico (nel caso in esame, il diritto) perché ci si trova di fronte al materiale grezzo, senza però lo strumento per elaborarlo. Il riferimento metodologico all'antropofagismo culturale si risolve quindi in un indirizzo di ricerca, in un invito a studiare anche

\footnotetext{
${ }^{17}$ Cf. il lungo paragrafo "Paulo Prado: o 'fautor' da Semana de 1922. Ao lado de sua intensa atividade como editor, organizador, mecenas e fomentador da arte moderna, Paulo Prado é autor de dois livros sobre aspectos sociais e culturais do Brasil, a partir da experiência colonial, publicados em momento de maturidade: Paulística: história de São Paulo (1925) e Retrato do Brasil: ensaio sobre a tristeza brasileira (1928). Trata-se, esta última, de uma obra que, segundo Oswald de Andrade, revelou aos brasileiros a 'existência' do Brasil (Andrade, O., 1929) ou, nas palavras de Mário de Andrade, 'fez papel de salva-vidas'; por outro lado, foi escrita em diálogo constante com o historiador Capistrano de Abreu (1853-1927), que Paulo Prado conhece por intermédio do tio, Eduardo Prado, e a quem ele chamará diversas vezes de 'Mestre”". In: WALDMAN, Thaís. À "frente” da Semana de Arte Moderna: a presença de Graça Aranha e Paulo Prado. Estudos históricos, Rio de Janeiro, vol. 23, jan./jun. 2010, n. 45. Disponível em: $<$ http://www.scielo.br/scielo.php?pid=S0103-21862010000100004\&script=sci_arttext>. Acesso em: 31 jan. 2015.
} 
gli eventi storico-giuridico brasiliani accanto alle teorie europee, e a studiarli in un contesto che estenda il campo d'indagine anche al materiale sociale, accanto all'indispensabile esegesi delle norme. Ma quale metodo usare nel seguire questo invito? È possibile trovarlo negli strumenti offerti dalla sociologia del diritto, dalla storia contemporanea del diritto o dalla sociologia storica, creando una sociologia storica del diritto?

Su queste constatazioni generali ritornerà il § 5, esaminando la ricostruzione dello sciopero ferroviario del 1906 secondo l'“antropofagia giuridica”. Ma prima è necessario esaminare il percorso metodologico attraverso il quale Siqueira giunge a quella ricostruzione.

\section{IL LIBRO DI GUSTAVO SIQUEIRA}

Alla luce di queste nozioni sul substrato culturale del libro (SIQUEIRA, 2014) si può ora passare a un rapido esame dei suoi primi tre capitoli, che sono intitolati: La storia del diritto prodotto dai movimenti sociali (esaminato qui di seguito alla lett. a); I movimenti sociali per la teoria e la metodologia della storia del diritto prodotto dai movimenti sociali (lett. b); Esperienze e antropofagie giuridiche per una storia del diritto plurale e problematizzante (lett. c). Invece al quarto capitolo, Esperienze giuridiche nelle strade ferrate: sciopero e cittadinanza nel 1906, è dedicato per intero il $\S 5$.

a) “La storia del diritto prodotto dai movimenti sociali”. Sin dalla lettura del titolo del volume si pone un quesito: la traduzione corretta è "storia del diritto prodotto dai movimenti sociali”, ovvero "storia del diritto nata dai movimenti sociali"; in altri termini, l'autore intende scrivere una storia del diritto che nasce dai movimenti sociali, oppure vuole scrivere sul diritto che nasce dai movimenti sociali? È fondamentale chiarire questa ambiguità perché l'idea insita in questa formulazione è la colonna vertebrale del volume.

Un conto è scrivere una storia dal punto di vista dei movimenti sociali, altro è scrivere una storia del diritto prodotto dai movimenti sociali. Infatti nel primo caso per 'diritto' si intende il diritto positivo, e se ne studia l'interazione con i movimenti sociali, mentre nel secondo caso si presuppone che i movimenti possano generare un ‘diritto' di cui si traccia la storia. Nel primo caso ci si richiama a una teoria formalistica del diritto, e per 'diritto' si intende il diritto positivo statuito e applicato secondo le regole di un testo costituzionale; nel secondo ci si richiama a una teoria antiformalistica del diritto, e allora per ‘diritto' si intendono le norme di comportamento sociale nate da una consuetudine intesa in senso lato. Il testo di Siqueira sembra oscillare fra le due 
concezioni, anche perché non definisce preliminarmente la nozione del ‘diritto’ di cui si traccia la storia: questa definizione (o, almeno, una precisazione) va ricercata nel contesto.

Nella pagina introduttiva, Siqueira definisce la "storia del diritto prodotto dai movimenti sociali" come "una teoria e metodologia della storia del diritto che utilizza le azioni dei movimenti sociali come uno degli elementi principali della ricerca storico-giuridica”, aggiungendosi "alle altre teorie e metodologie della storia del diritto, cercando di costruire una storia critica e problematizzante”. La "storia del diritto" si presenta quindi come "insieme di esperienze giuridiche esistente in un certo periodo, mentre la "storia del diritto prodotto dai movimenti sociali" si presenta “come uno strumento di comprensione, di critica e di problematizzazione della storia del diritto”. In questo contesto, lo sciopero ferroviario del 1906 "mostra come le varie tensioni di quel periodo possano contribuire alla comprensione di quello che era il diritto di sciopero e le sue negazioni in quell'importante momento storico del Brasile” (p. VIII). Esistono quindi delle norme giuridiche positive, e l'autore propone di studiare, nei movimenti sociali, i comportamenti ad esse in qualche modo riconducibili: quello che propone sembra quindi avvicinarsi a una sociologia storica del diritto.

Infatti i punti di partenza dell'analisi sono testi sociologici, soprattutto di Pierre Bourdieu $(2001)^{18}$ e di António Manuel Hespanha (2005, 2007), cioè d’un sociologo e d'uno storico del diritto aperto a una visione sociologizzante e alternativa del diritto. Da essi Siqueira (2014) ricava la direttrice secondo cui “il diritto e le sue esperienze nell'esistenza non possono essere ridotte alla

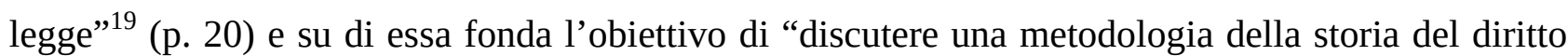
che riesca a conoscere non soltanto la legge - come in certo modo propone la storiografia positivista-legalista della storia del diritto - ma una metodologia che sia sensibile alla pluralità delle manifestazioni giuridiche, che possa conoscere le tensioni, le contraddizioni del diritto nella società umana”, cioè che "sia aperta in ogni momento ad altri elementi che possano integrare la storia del diritto” (p. 20). Come afferma Hespanha, “ogni società ha più norme che quelle solo giuridiche”: la proposta metodologica si muove dunque nell'ambito dell'antiformalismo giuridico ovvero del pluralismo giuridico, che "rompe i rapporti con il feticcio delle leggi e delle idee giuridiche come unici elementi che spiegano la storia del diritto" (p. 21) e va alla ricerca di "altre esperienze giuridiche diverse da quelle tradizionalmente studiate dalla storia del diritto” (p. 23). Si tratta cioè

\footnotetext{
${ }^{18}$ Citato nella traduzione BOURDIEU, Pierre. O poder simbólico. Rio de Janeiro: Bertrand Brasil, 1989, soprattutto le p. 212-216 e p. 243.

19 Nell’originale è usato il termine “lei” (legge), e non “direito” (diritto). L'uso dei due termini in portoghese corrisponde all'uso italiano della coppia equivalente.
} 
di vedere se "certe azioni politiche che non sono considerate giuridiche" in certi casi "possono contenere elementi di giuridicità” (p. 27).

Una di queste esperienze diverse è "la discussione sullo sciopero del 1906, nella quale sarà possibile comprendere il trattamento da criminali riservato al movimento degli scioperanti, anche se esso esercitava un diritto consacrato dai tribunali e dalla dottrina” (p. 23) e fondato sul diritto positivo, come si vedrà: ma allora questa storia del diritto come può evitare il confronto anche con il diritto positivo? Siqueira spiega che "la storia del diritto attraverso i movimenti sociali è una disciplina che deve camminare a fianco delle altre: ha bisogno della storia dei concetti, della storia delle idee, della storia dogmatica e della storia sociale” (p. 25). È probabile che con "storia dogmatica” l'autore intenda la storia del diritto positivo tradizionale, quella cioè che egli non desidera praticare ma di cui deve inevitabilmente tenere conto nella costruzione di questa metodologia complessa, plurale, critica, che non vuole "semplicemente negare o decostruire le altre metodologie, ma arricchire il dibattito con un argomento nuovo” (p. 25). Si mira quindi a un “pluralismo metodologico" (p. 26), con aperture all'antropologia e alla sociologia del diritto "che possono aprire gli occhi del giurista su realtà prima non percepite” (p. 27).

Il punto è se "la giuridicità può essere incontrata al di fuori delle norme positive” (p. 28). Forse bisognerebbe distinguere nettamente tra fonti del diritto e fonti della storia del diritto: ma prima ancora, proprio per delimitare con precisione il campo d'azione, ritengo che bisognerebbe definire esplicitamente che cosa si intende per 'diritto', perché da ciò deriverebbe con precisione quali sono le fonti da prendere in considerazione per tracciare una 'storia del diritto'.

Siqueira (2014, p. 28) ammette il pluralismo giuridico:

Se le fonti del diritto sono tradizionalmente ricollegate allo Stato come unico produttore del diritto e l'ipotesi qui discussa è un ampliamento di queste fonti, verificando che si può trovare la giuridicità al di fuori delle norme positive, sarà fondamentale discutere il rapporto tra le fonti della storia del diritto e il pluralismo giuridico. ${ }^{20}$

Siqueira propone anche un uso più esteso delle tradizionali fonti della storia giuridica, che “possono essere usate anche per conoscere una 'storia marginale', verificando per esempio quanto si possano usare gli archivi delle prigioni, dei commissariati, dei processi penali per conoscere una 'mentalità marginale'”' (p. 29). Resta però da vedere quanto una ricerca di questo genere sia storicogiuridica o piuttosto storico-sociologica.

\footnotetext{
${ }^{20}$ Questa concezione si ricollega direttamente a António Hespanha (cit., 2007, p. 524) e a Boaventura de Sousa Santos (SANTOS, Boaventura de Sousa. O discurso e o poder. Ensaio sobre a sociologia da retórica jurídica. Porto Alegre: Fabris, 1988).
} 
In conclusione, “il pluralismo permette di percepire l'azione dei movimenti sociali come azioni giuridiche di lotta per il diritto e per la cittadinanza” e i movimenti sociali divengono così “soggetti del diritto" e si possono interpretare "le sue azioni come azioni giuridiche e non solo come azioni devianti (marginalizadas) o criminalizzate” (p. 30). ('Marginal' in portoghese indica in generale 'chi sta al margine della società' e quindi, più specificamente, il 'deviante'). Il “diritto prodotto dai movimenti sociali" coesiste quindi col diritto positivo: “Questo pluralismo non esclude il diritto ufficiale, formale” (p. 31).

I passi fin qui citati lasciano aperta un’ambiguità di fondo che pervade l'intera ricerca: non v’è dubbio che un'azione “criminalizzata” è un'“azione giuridica” (cioè essa è oggetto dell'applicazione del diritto positivo preesistente). Resta da dimostrare - e in questa direzione la proposta di Siqueira esprime più un desiderio che una realizzazione - se e in che misura $\mathrm{i}$ movimenti sociali creano norme vincolanti per la comunità da cui esse scaturiscono (cioè se essi sono soggetto creatore di un diritto positivo nuovo).

"Per la storia del diritto prodotto dai movimenti sociali, il diritto è ciò che le persone sentono come diritto” (p. 32). Questo approccio non formalista può essere ricondotto a discipline diverse: alla psicologia giuridica, per esempio, o al “diritto trovato per strada”21 (p. 33) o alle varie forme di diritto libero o alternativo. Siqueira si distacca però da questi modelli: per lui, "il diritto trovato per strada” ha un oggetto limitato (i movimenti brasiliani degli anni Settanta) e “una prospettiva di sociologia giuridica con una considerazione critica del diritto attuale”, mentre la sua proposta metodologica si estende ad altri movimenti sociali, vuole "comprendere le esperienze giuridiche passate e il loro accostamento al presente”, non limitandosi appena "al secolo XX” (p. 33). Analogamente Siqueira prende le distanze dal diritto libero in forma troppo sintetica e non argomentata, a mio giudizio incompatibile con le vaste e inevitabili analogie tra la sua proposta e quell’approccio non formalistico: la sua indagine sul “diritto e percezione di ciò che è diritto”, afferma in una nota, non intende "teorizzare su una Scuola del Diritto Libero come fa Hermann Kantorowicz, ma aprire la ricerca storica alle diverse manifestazioni del diritto” (p. 34, nota 78).

In conclusione, la "storia del diritto prodotto dai movimenti sociali” intende differenziarsi dalla sociologia del diritto e dai movimenti antiformalistici (Movimento del diritto libero, diritto alternativo e teorie analoghe).

\footnotetext{
21 Il movimento del "direito achado na rua” dagli anni Settanta cerca di conciliare il diritto con le esigenze del proletariato urbano e agrario. Iniziato da Roberto Lyra Filho (1926-1986), produsse la Nova Escola Jurídica Brasileira e la sua rivista "Direito \& Avesso" (Diritto e rovescio). La sua vasta produzione giuridica è direttamente collegata con i movimenti sociali degli anni Settanta e Ottanta.
} 
b) "I movimenti sociali per la teoria e la metodologia della storia del diritto prodotto dai movimenti sociali”. Il capitolo successivo del volume (dal titolo veramente oscuro) si propone di delimitare a grandi linee, più che definire puntualmente, la nozione di 'movimento sociale' di cui Siqueira fa uso per studiarne dal punto di vista storico i rapporti con il mondo giuridico. "I movimenti sociali sono eventi (ocorrências) umani in cui le persone, condividendo esperienze comuni, agiscono emergendo da processi storici che possono essere studiati soltanto in un certo periodo storico” e che non si limitano a una specifica classe sociale (p. 56). Questa visione si rifà soprattutto all’opera di Maria Gohn (1997, 2009) (la cui lettura ritiene “essenziale”), oltre che al già ricordato Bourdieu, a Ilse Scherer-Warren (1993), a Eric Hobsbawm (2001) e all'edizione in portoghese del Dizionario di politica curato da Bobbio e altri. "L’identità dei movimenti” "non è determinata da un 'essere’”, bensì da “un 'agire”” (p. 59) e da un "sentire” (p. 60); deve “manifestare almeno parte dei suoi obiettivi”, che devono avere "una connotazione politica” (p. 65); inoltre i movimenti possono essere popolari, ma anche settoriali o elitari, ovvero misti (p. 70); infine, un movimento esige "un minimo di organizzazione” (p. 63). La realtà cui Siqueira rivolge la sua attenzione è quindi eterogenea: può essere classista o non classista, può operare in uno o più luoghi, mentre la sua azione può essere attiva o passiva, ovvero contemporanea o storica; l'unico limite è l’impossibilità di “un movimento sociale che contenga una sola persona” (p. 63).

“L’obiettivo della storia del diritto prodotto dai movimenti sociali è riuscire a percepire queste esperienze, siano esse politiche o giuridiche”; ed è politica “un’azione che mira a intervenire nelle strutture e nelle istituzioni sociali”22; il che, seguendo Maria Gohn, consente di "percepire i movimenti sociali come ‘espressioni del potere della società civile’ e come ‘processi politicosociali', le cui azioni, in certi momenti, possono contenere esperienze giuridiche importanti per la comprensione della storia del diritto” (p. 63).

Di fronte a questa eterogeneità, si pone il problema di individuare "quando le azioni dei movimenti sociali possono essere intese come azioni giuridiche”, in base a una nozione di diritto fin qui evocata solo intuitivamente (p. 65 et seq.). Queste azioni sociali “sono giuridiche quando hanno una relazione con qualsiasi esperienza giuridica”, cioè “con il diritto, con le leggi (mettendo in discussione la loro positivizzazione, o violandola o asserendola), con i tribunali, con il senso della giustizia e con una pluralità di norme” (p. 66). Questo oggetto d’indagine, a mio giudizio, viene a coincidere con quello della sociologia storica o giuridica, ovvero con entrambe; o anche con l’oggetto della scienza politica, come precisa lo stesso Siqueira: “Queste azioni sono anche politiche, perché i movimenti sociali spesso anticipano i conflitti sociali che saranno poi discussi dal

\footnotetext{
${ }^{22}$ Definizione mutuata da Hespanha (1978, p. 31).
} 
diritto" (p. 66). Infatti, per Siqueira, "il sentimento del giuridico può esistere anche al di fuori del diritto positivo", intendendo quest'ultimo nel senso corrente di diritto "positivizzato dagli organi dello Stato” (p. 67). È di questo diritto fuori dal diritto che Siqueira propone di tracciare la storia.

I due esempi addotti per illustrare questo sentimento del giuridico esistente anche al di fuori del diritto positivo suscitano qualche dubbio. Il primo è desunto dalle lotte operaie del secolo XIX: quando gli operai londinesi rivendicarono "il diritto a votare un membro del parlamento, non si fondavano sul diritto positivo, bensì su una percezione sociale, culturale e storica di ciò che per loro, in quel momento, era diritto” (p. 68). Rivendicavano cioè un diritto non ancora esistente, non ancora positivo. Diverso mi pare invece il caso dello sciopero ferroviario del 1906, che Siqueira anticipa qui e che tratterà poi per esteso nel quarto e ultimo capitolo (cfr. § 5). Quei ferrovieri “credevano di possedere, al di là del diritto positivo, un diritto di sciopero: avevano una percezione sociale, culturale e storica di quello che era il loro diritto in quel momento” (p. 68). Però i ferrovieri non rivendicavano la positivizzazione di un diritto cui aspiravano, ma chiedevano l'applicazione di una norma positiva che riconosceva formalmente il diritto di sciopero, ma che di fatto veniva disapplicata. A mio giudizio, i due esempi sono radicalmente diversi. Hanno però un elemento in comune, che Siqueira mette jheringhianamente il luce così: "La lotta per il diritto non positivo, ma riconosciuto culturalmente, socialmente e storicamente è anche una lotta giuridica”. Ma la storia di questa lotta è storia giuridica?

Ogni sciopero è una lotta per rivendicare un diritto - già statuito ma non applicato, oppure ancora da statuire - però è una lotta in cui l'elemento giuridico viene alla fine, quasi come l'appendice normativa d'una vittoria sindacale. Un esempio estremo è lo sciopero ferroviario rievocato dallo scrittore della DDR Bodo Uhse (1956, p. 94) nel suo incontro in Cina con un ferroviere che aveva partecipato a tutta l'epopea rivoluzionaria cinese e che gli "parla dei tempi passati, quando la ferrovia Pechino-Hankau era sotto amministrazione francese: dieci ore di lavoro al giorno, venti centesimi di paga e per ogni sbaglio una trattenuta di cinquanta centesimi, cioè un giorno e mezzo di paga. Ormai fa parte della storia quello sciopero del 7 febbraio 1923, con cui i lavoratori di Hankau cercarono di opporsi a quello sfruttamento”. Intervenne l'esercito, alcuni scioperanti vennero uccisi, altri (come il narratore) passarono alla clandestinità. Nel 1949 l'Esercito Popolare di Liberazione entrò in Hankau e, nel stesso luogo in cui gli scioperanti "erano stati uccisi a sciabolate, il colpevole Monsieur Durocs, amministratore della ferrovia Pechino-Hankau, venne giustiziato” (UHSE, 1956, p. 94). Sarebbe possibile trasformare questa narrazione letterariorivoluzionaria in un capitolo di storia del diritto? Ecco la sfida metodologica insita nella proposta di Siqueira. 
Al di là di questi problemi di storia del diritto positivo, l'aspetto più problematico d'una concezione non formale del diritto è insito nel rischio di legittimare ogni movimento sociale, e quindi ogni diritto che da esso derivi. È un tema ricorrente in Brasile, dove è diffusa una concezione anti-formalistica del diritto che si richiama al diritto alternativo o all'uso alternativo del diritto $\left(\right.$ LOSANO, 2007) ${ }^{23}$. I movimenti antiformalistici tendono a identificare le richieste popolari con i valori progressisti, democratico-parlamentari o di sinistra (cioè egualitari). Però non bisogna dimenticare che tanto il nazionalsocialismo quanto il fascismo furono anche movimenti di massa e che la loro visione del diritto come subordinato alla politica portò alla distruzione dello Stato di diritto. Il positivismo giuridico dà almeno la certezza del diritto ${ }^{24}$.

Anche la concezione della "storia del diritto prodotto dai movimenti sociali" solleva problemi analoghi, soprattutto ai giorni nostri, in cui - di fronte alla crisi dei partiti politici tradizionali - si moltiplicano i movimenti ideologicamente ambigui o decisamente di destra ${ }^{25}$.

\begin{abstract}
È nella cultura che il diritto è percepito dai gruppi sociali ed è trasformato in oggetto di lotta. Lotta che avviene in una certa società, in un certo periodo storico. Per questo si ritiene che i movimenti sociali siano elementi fondamentali per una storia del diritto, perché essi possono rendere percepibili anche le lotte per i diritti non positivi e le esperienze giuridiche provenienti da un altro settore della vita sociale. La storia del diritto prodotto dai movimenti sociali rende possibile la percezione di altri diritti (SIQUEIRA, 2014, p. 69).
\end{abstract}

In questa frase, il termine 'diritto' è usato in senso così lato, che si presta a contenere oggetti eterogenei e incompatibili.

Nel momento in cui un parlamento legifera, trasforma in legge una scelta politica accettata dalla maggioranza o dall'oligarchia al potere. Definire questa scelta come fondata su un "preconcetto" implica un giudizio di valore negativo sulla norma così positivizzata, e quindi un

\footnotetext{
${ }^{23}$ In questo libro ho esaminato questi problemi posti dalla teorie antiformalistiche del diritto. In particolare, il paragrafo L'uso alternativo del diritto in Brasile (p. 253-8) affronta il problema dei rapporti fra diritto e movimenti di destra. Cf. inoltre LOSANO, Mario G. La legge e la zappa: origini e sviluppi del diritto alternativo in Europa e in Sudamerica. Materiali per una storia della cultura giuridica, ano 30, n. 1, Bologna, Il Mulino, 2000. Tradução para o espanhol, por Andrea Greppi, disponível em: <http://e-archivo.uc3m.es/bitstream/10016/1384/1/DyL-2000-V-8-Losano.pdf>. Acesso em: 31 jan. 2015. La traduzione in spagnolo è ripresa in CARVALHO, Amilton Bueno de; Carvalho, Salo de (Org.). Direito alternativo brasileiro e pensamento jurídico europeu. Rio de Janeiro: Lumen Juris, 2004.

${ }^{24}$ La certezza giuridica non è sempre politicamente accettabile. Se un movimento di destra porta al potere una dittatura, questa emanerà il suo diritto, contro il quale il positivismo giuridico non avrà nulla da obiettare perché rifiuta di giudicare il diritto positivo secondo valori extragiuridici. È questa una delle accuse rivolte a Hans Kelsen. Ma in questa sede è possibile solo accennare a questo capitale problema. Una sintesi dell'itinerario anche giuridico d'una presa di potere è in BROSZAT, Martin. Da Weimar a Hitler. Roma: Laterza, 2001.

${ }^{25}$ L'appassionante tema dei movimenti sociali attuali non può essere qui sviluppato, ma ne avevo trattato nel novembre 2013 nel seminario Movimentos e política na Europa contemporânea, organizzato da Siqueira nell’Universidade do Estado do Rio de Janeiro (UERJ) e articolato in quattro temi: Movimentos e partidos na Europa do terceiro milenio; "Indignados" na Espanha, "Piraten" na Alemanha; O "Movimento 5 Estrelas" na Itália; Qual a compatibilidade entre movimentos e democracia parlamentaria? Il 2015 si è aperto in Germania con il movimento Pegida ("Patrioti Europei contro l'Islamizzazione dell'Occidente") che sta suscitando vari contro-movimenti, in un clima di generale opacità ideologica.
} 
preconcetto positivo sul movimento sociale che vi si oppone in qualche maniera. "Spesso, in base a un preconcetto (preconceituosamente), sono positivizzate come illecite condotte popolari o manifestazioni contrarie allo status quo sociale” (p. 69): e Siqueira cita "la criminalizzazione degli scioperi, delle marce, delle associazioni di capoeira o religiose", viste talora "come illecite o devianti (marginais)” perché “non contribuiscono al ‘buon’ andamento della società” (p. 69).

In questa presa di posizione a favore dei movimenti (indipendentemente della nozione di diritto da cui si può partire) è implicito un giudizio di valore positivo, come si è detto poco fa. Ma ci sono anche movimenti antidemocratici. Indubbiamente, "dichiarate devianti o no, queste azioni politiche possono favorire la comprensione delle esperienze giuridiche di una certa epoca” (p. 69). Ma lo studio scientifico di queste “azioni politiche” è giuridico o sociologico?

c) "Esperienze e antropofagie giuridiche per una storia del diritto plurale e problematizzante". Definita la natura e il campo di azione dei movimenti sociali, per precisare il concetto di “esperienza” più volte richiamato nel descrivere l'azione "giuridica” dei movimenti stessi ${ }^{26}$, viene introdotto il concetto di “antropofagia, nell’uso politico attribuitogli da Oswald de Andrade”, perché esso "può contribuire a spiegare questa pluralità di esperienze giuridiche” fin qui illustrata (p. 76).

Il termine di esperienza è “usato come sinonimo di ‘vissuto' (vivencia), affinché sia chiaro che tanto il vissuto quanto le esperienze sono molteplici” (p. 77). L'ampia analisi del concetto di esperienza mette a confronto autori soprattutto italiani - come Giuseppe Capograssi (1959, p. 10-2; 1937, p. 38, p. 224), Guido Fassò (1953, p. 12, p. 96 et seq.), Paolo Grossi( 2010, p. 142, p. 153), Enrico Opocher (1983, p. 16 et seq.), Widar Cesarini Sforza (1958, p. 65, p. 108) - ma anche Reinhart Kosellek (2006, p. 309, p. 311) e Miguel Reale (1968, p. 6, p. 34, p. 47, p. 128, p. 145, p. 218 et seq.).

Con questi autori Siqueira non sempre concorda (p. 91). Respinge anche le visioni dello Stato come “unità, integralità dell’esperienza giuridica” perché esso “disprezza i valori devianti, paralleli e non maggioritari” (p. 92). La metodologia della storia del diritto prodotto dai movimenti sociali vuole invece esplorare "i lati oscuri, le violenze non dette, le esperienze fatte in silenzio, $\mathrm{i}$ mondi occulti nei sotterranei della storia” (p. 95). In essa "le scienze dialogano: l'antropologia, la sociologia e la storia del diritto si mescolano, infrangono barriere e problematizzano ogni volta di più le realtà umane” (p. 97). Ecco giunto il momento dell'antropofagia giuridica e del ritorno ai temi cari al testo di Oswald de Andrade e al mondo culturale del § 3.

\footnotetext{
${ }^{26}$ Cf. SIQUEIRA, Gustavo Silveira. Pensamiento y norma. La contribución del concepto de experiencia jurídica para la historia del derecho. In: FERRARI, Anderson et al. Horizontes de Brasil. Barcelona: APEC, 2011, p. 1440-5.
} 
Siqueira tenta ora "una piccola sovversione, questa volta del testo di Andrade", proponendo di costruire un’antropofagia giuridica, cioè “digerire criticamente il diritto” (p. 99). Infatti, “per costruire qualsiasi teoria critica e problematizzante del diritto, è necessario deglutire i concetti, le storie, le teorie, ma con una coscienza critica. Non basta inghiottire: bisogna digerire. [...] Attraverso questo processo nasce il nuovo, il ri-creato, l’antropofagico” (p. 100). Per questo “il diritto è più delle leggi positive, e le sue storie hanno bisogno di esser sempre oggetto di antropofagia”, perché

nel diritto, l'antropofagia diviene l'esigenza di non limitarsi a inghiottire le teorie e le dottrine nazionali (e straniere), ma problematizzarle, criticarle, pensarle in funzione del mondo in cui si vive. [...] È tentar di collocare il diritto nell'alterità, nell'intimità con la società, il che può anche trasformarsi in autocritica del diritto, in autofagia della sua stessa essenza (SIQUEIRA, 2014, p. 101).

Questa visione dell' “antropofagia giuridica dialoga con le teorie della recezione perché il discorso cessa di essere inteso soltanto nel senso desiderato dall'autore, e diviene opera anche del lettore" ed ha il suo manifesto, parallelo a quello di Andrade, nella Letteratura come provocazione del romanista Hans Robert Jauss (1993, p. 102): “La volontà dell’autore (e del legislatore, nel caso giuridico) perde d'autonomia a favore d'una realtà che batte alla porta e rinfresca i testi”. Quindi

l'antropofagia giuridica permette al ricercatore di problematizzare i metodi e le teorie utilizzati per far ricerca e di discuterne l'influenza nell'analisi delle esperienze giuridiche e nei risultati delle ricerche. Permette di pensare le teorie in funzione del Brasile, invece di pensare il Brasile in funzione di queste teorie (SIQUEIRA, 2014, p. 104).

In realtà, nell’aprile del 1967 Jauss presentò la sua lezione magistrale con un titolo più tradizionale, ma il suo nucleo (ripreso anche in scritti successivi) era chiaro: l'opera letteraria "è come uno spartito, che si rimette (angelegt) a una sempre rinnovata risonanza della lettura, che libera il testo dalla materia delle parole e che lo conduce alla sua esistenza attuale” (JAUSS, 1967, p. 30$)^{27}$. Non si può qui neppure accennare al quesito se (e, eventualmente, sino a che punto) le raffinate costruzioni che nascono da questo capovolgimento della visione tradizionale possano essere trasferite dalla teoria letteraria a quella giuridica, cioè dall'opera d'arte al testo normativo. Non a caso le frasi di Siqueira sopra citate richiamano la teoria del diritto, e non la sua prassi. Tanto un romanzo quanto una legge sono testi verbali e, in quanto tali, "they are worthy of study essentially because they can be read and can engender responses in human beings" (SLATOFF, 1970, p. 3). Ma una risposta negativa genera una conseguenza estetica nel romanzo e una

\footnotetext{
27 Tema ripreso più ampiamente in JAUSS, Hans Robert. Literaturgeschichte als Provokation. Frankfurt am Main: Suhrkamp, 1970.
} 
conseguenza pratica nella norma, perché quest'ultima è accompagnata da una sanzione ${ }^{28}$. Indubbiamente esistono alcuni punti di contatto: lo stesso testo delle norme brasiliane sul diritto di sciopero ha generato letture differenti nei politici e nei ferrovieri.

Il richiamo di Siqueira a Jauss che evoca i rapporti tra letteratura e diritto non può essere affrontato, perché questi rapporti hanno ormai generato un nuovo settore degli studi giuridici. Un'altra chiave di lettura - anche questa solamente accennata, e non presente in Siqueira - potrebbe venire dalla teoria dell'implementation, cioè dalle tecniche sociologico-organizzative che studiano di quali effetti previsti o non previsti, desiderati o non desiderati può produrre una norma giuridica. Ma conviene qui chiudere questa digressione e vedere come l' “antropofagia giuridica” ha digerito lo sciopero ferroviario del 1906.

\section{L’ANALISI STORICO-GIURIDICA DELLO SCIOPERO FERROVIARIO DEL 1906}

Dopo il dibattito metodologico sin qui analizzato è venuto il momento di esaminare il fatto cui applicare il metodo proposto, cioè lo sciopero ferroviario del 1906. I punti da esaminare sono tre.

In primo luogo (lett. a) si deve esaminare la legislazione vigente al momento dello sciopero, per stabilire se la legge lo permetteva o no, e quindi per capire se gli scioperanti rivendicavano l'approvazione di un diritto non ancora esistente oppure l'applicazione di un diritto positivo, ma disatteso. Questo approccio ricade nella storia della legislazione in senso stretto, più che in una storia del diritto in senso lato, ma è il presupposto indispensabile per l'ulteriore svolgimento della ricerca.

In secondo luogo (lett. $b$ ) si deve esaminare lo svolgimento dello sciopero stesso, poiché per la maggioranza dei lettori è indispensabile la ricostruzione fattuale di un evento così lontano nello spazio e nel tempo. Questo approccio ricade nella storia sociale o nella sociologia storica del diritto.

In terzo luogo (lett. c) si deve esaminare in che modo il metodo "antropofagico" viene applicato a questo insieme normativo e fattuale, argomento trattato nel quarto e ultimo capitolo del libro di Gustavo Siqueira (pp. 112-159). Questo approccio mette alla prova in che misura il metodo “giuridico-antropofagico" contribuisce alla costruzione di una "storia del diritto prodotto dai movimenti sociali”, come indica il titolo del volume di Siqueira.

\footnotetext{
28 “L'opera letteraria possiede due poli, che si potrebbero chiamare il polo artistico e e il polo estetico: quello artistico indica il testo creato dall'autore e quello estetico la concretizzazione fornita dal lettore”. Cf. ISER, Wolfgang. Der Akt des Lesens. München: Fink, 1976, p. 38.
} 
a) Le norme: il diritto di sciopero nel Brasile repubblicano. La dinastia dei Bragança, che reggeva il Brasile dalla sua indipendenza nel 1822, abolì la schiavitù con la "Legge aurea” del 1888. Questa misura colpì economicamente $\mathrm{i}$ grandi proprietari terrieri, detentori del potere reale, che appoggiarono il passaggio alla repubblica. Anche in questa transizione la storia del Brasile è peculiare, perché nel 1889 una monarchia illuminata e costituzionale venne sostituita da una repubblica conservatrice e presidenzialista.

Concentrando l'attenzione sul diritto di sciopero, il punto di partenza obbligato è il codice penale del 1890 che, nella sua formulazione originaria e sulla scia del codice portoghese del 1886, negava il diritto di sciopero agli articoli 204, 205 e 206, qui di seguito tradotti (p. 113):

Capo VI - I reati contro la libertà di lavoro.

Art. 204. Il costringere, o l'impedire ad alcuno di esercitare la sua industria, commercio o professione; di aprire o chiudere i suoi stabilimenti, officine e sedi; di lavorare o di omettere di lavorare in certi giorni determinati, è punito con la detenzione da uno a tre mesi.

Art. 205. Il convincere, o l'alliciar operai e lavoratori affinché abbandonino gli stabilimenti in cui sono impiegati, con la promessa d'una ricompensa o sotto la minaccia d'un danno, è punito con la detenzione da uno a tre mesi e con la multa da 200 a 500.000 contos $^{29}$.

Art. 206. Il causare o il provocare la fine o l'interruzione del lavoro, per imporre agli operai o un aumento o una riduzione di orario o di salario, è punito con la detenzione da uno a tre mesi.

c. 1. Se gli interessati si uniscono a questo fine, la pena per i capi dell'organizzazione è la detenzione da due a sei mesi.

c. 2. Se c'è ricorso alla violenza, la pena è la detenzione da sei mesi a un anno, in aggiunta alla pena in cui si incorre per la violenza.

Queste norme che proibivano lo sciopero pacifico provocarono un'ondata di proteste, anche perché l’inizio del Novecento vide nascere in Brasile i primi movimenti e partiti operai. Questi movimenti protestavano contro un diritto vigente e chiedevano l'emanazione d'una nuova normativa. Circa due mesi dopo la promulgazione del codice penale, ma ancora durante la sua vacatio, gli articoli 205 e 206 vennero modificati dal decreto 1162 del 12 dicembre 1890, che li riformulava in questi termini (p. 114):

Il Capo del Governo Provvisorio della Repubblica degli Stati Uniti del Brasile, considerando che la redazione degli artt. 205 e 296 del Codice penale nell'applicazione può suscitare dubbi e interpretazioni erronee, al fine di stabilire la chiarezza indispensabile soprattutto nelle leggi penali, decreta:

Art. 1. Gli artt. 205 e 206 del Codice Penale vengono così formulati:

Arti. 205. L’allontanare con minacce e coazione gli operai e i lavoratori dai luoghi in cui sono impiegati, è punito con la detenzione da uno a tre mesi e con una multa da 200 a 500.000 contos.

\footnotetext{
${ }^{29}$ La valuta brasiliana era il real (pl. réis); “conto de réis” (e solo "contos”) corrispondeva a un milione di réis. È difficile stabilirne il controvalore attuale, ma la multa indicata nella legge rappresentava una somma ingente, specie se rapportata a un salario operaio all'inizio del Novecento.
} 
Art. 206. Il causare o il provocare la cessazione o l'interruzione del lavoro con minacce o violenza, per imporre agli operai o ai padroni un aumento o una diminuzione di orario o di salario, è punito con la detenzione da uno a tre mesi.

Grazie a questo emendamento, alla soglia degli eventi del 1906 la situazione giuridica era dunque la seguente: lo sciopero era punito se provocato con la violenza, mentre lo sciopero pacifico cessava di essere un reato. I testi dottrinari citati da Siqueira concordano su questa conclusione ${ }^{30}$.

Infine, dal punto di vista della storia giuridica è centrale il ricorso degli avvocati degli scioperanti al Tribunal de Justiça di São Paulo, perché esso dimostra come le autorità pubbliche procedessero con la mano troppo pesante contro gli scioperanti e chi stava dalla loro parte. Gli avvocati degli arrestati dovevano recarsi a Jundiaí il 19 maggio per difenderli, ma la polizia di São Paulo impedì loro di salire sul treno. Essi presentarono perciò una richiesta di habeas corpus, che venne accolta dalla magistratura e, con questa sentenza, partirono il 20 maggio per Jundiaí, dove però la polizia impedì loro di scendere dal treno, cosicché dovettero tornare a São Paulo.

Il 21 maggio il Tribunal de Justiça di São Paulo respinse una nuova impugnazione degli avvocati, adducendo l'incompletezza della documentazione. Il parere di minoranza si richiama esplicitamente alle norme già esaminate: "I reati contro la libertà del lavoro [...] non comprendono lo sciopero pacifico”; “quello che la legge punisce sono gli attentati alla libertà di lavoro” (p. 133). Infine il ricorso al Supremo Tribunal Federal (cioè alla Corte di Cassazione brasiliana) diede ragione agli avvocati degli scioperanti con una decisione del 9 giugno 1906. Però il 26 maggio il servizio ferroviario era ripreso normalmente e quindi la sentenza del Supremo Tribunal Federal offriva una soddisfazione soltanto morale agli scioperanti.

La scelta metodologica pone ora Siqueira davanti a un bivio: i manuali espongono, egli afferma, “come questa legislazione era intesa dal giudiziario, dall'esecutivo, dalla dottrina e da una parte della società”; la sua opzione per il metodo giuridico-antropofagico lo porta invece a “verificare come il diritto di sciopero era esercitato dai movimenti sociali” (p. 116).

b) I fatti: lo svolgimento dello sciopero ferroviario del 1906. All'inizio del secolo XX il caffè era il principale prodotto agricolo esportato dal Brasile. Le due imprese ferroviarie che trasportavano il caffè dalle zone di produzione, nell'interno degli Stati di São Paulo e di Minas Gerais, fino alla costa e, in particolare, al porto di Santos, erano la Companhia Paulista e la Companhia Mogyana. Entrambe avevano sede a Campinas, una città vicina a São Paulo. Lo sciopero del 1906 coinvolse

\footnotetext{
${ }^{30}$ Cf. MORAES, Evaristo de. Apontamentos de direito operário. Ed. fac-sim. São Paulo: Ltr, 1998; SIQUEIRA, Galdino. Direito penal brasileiro. 2. ed. Rio de Janeiro: Jacyntho, 1932, 2 v.; HUNGRIA, Nelson. Compêndio de direito penal. Rio de Janeiro: Jacyntho, 1936.
} 
dapprima la Companhia Paulista, e si estese poi anche alla Companhia Mogyana e ad altre imprese non ferroviarie.

Lo sciopero ferroviario iniziò il 15 maggio 1906, nel mese cioè della raccolta del caffè. Ma una debolezza del testo di Siqueira è che, a questo punto della lettura, non viene descritto con chiarezza lo svolgimento dello sciopero, mentre vengono esposte le prese di posizione soprattutto degli scioperanti. Si analizzano cioè i giudizi di valore su fatti enunciati in modo frammentario.

Lo sciopero fu un atto di solidarietà con lo sciopero della conceria di Jundiaí (città dello Stato di São Paulo), iniziato con le "proteste contro il capostazione di Jundiaí” (p. 125): però manca l'esposizione dei fatti che provocarono le proteste. Solo dopo una dozzina di pagine si apprende che gli operai della Companhia Paulista rivendicavano "la rimozione del capostazione che violava la dignità degli operai” (p. 136) e che per questa ragione il 19 maggio 1906 gli operai della Companhia Mogyana iniziarono uno sciopero di solidarietà con i colleghi paulisti.

Solo due lunghe note a pié di pagina, a questo punto, riassumono i fatti desumendoli da una ricerca di Boris Fausto. La Companhia Paulista aveva intrapreso "una politica di modernizzazione che, nella sua forma classica, intaccava il livello di impiego e la retribuzione degli operai, accanto a misure che limitavano l’organizzazione autonoma di questi ultimi”. Nell’applicare queste misure, l'ingegnere Monlevade (che sembra essere non un capostazione, ma "l’ingegnerecapo del materiale rotabile [chefe de locomoção] della compagnia”) si fece notare per "il trattamento rude nel contatto personale con gli operai”, per le riduzioni salariali e per i licenziamenti, nonché per aver imposto l’iscrizione obbligatoria all'“associação beneficiente”, cioè alla mutua aziendale di cui gli operai criticavano le cattive prestazioni. Infine, secondo Boris Fausto (1977, p. 135), “l’attrito provocato dall'ingiusto trasferimento di un funzionario è il detonatore di un movimento di 3800 lavoratori” ${ }^{31}$.

Il differente approccio metodologico spiega la critica di Siqueira alla descrizione fattuale di Fausto, che a suo giudizio "si occupa poco delle varie manifestazioni che adducevano la violazione della dignità dei lavoratori come uno dei motivi fondanti del movimento degli scioperanti” (p. 136, nota 328). Per Siqueira è invece fondamentale il fatto che gli scioperanti rivendichino la propria dignità (e qui si manifesta ancora una volta la sua concezione particolarmente lata del diritto): “L’argomentazione degli scioperanti è giuridica. Esigono la dignità inerente a ogni persona, così come lottano per quello che è giusto e ritengono un loro diritto” (p. 125).

In realtà, le due argomentazioni - rivendicazioni economiche e tutela della dignità operaia - non si escludono, ma sono complementari: quello che provoca lo sciopero sono i fatti, vissuti

\footnotetext{
${ }^{31}$ Citato da Siqueira a p. 136, note 327 e 328.
} 
come lesivi della dignità operaia. Le modalità dei trasferimenti dovettero essere infatti decisamente offensive, se provocarono uno sciopero che - partendo dal licenziamento di un funzionario: evidentemente la goccia che fece traboccare il vaso - coinvolse quasi quattromila persone provenienti da imprese d’ogni settore nell’intero Stato di São Paulo.

Un fatto sicuro, nota Siqueira, è che "in pochi giorni lo sciopero causò disturbi immensi all'economia del paese. I sacchi di caffè non vennero più inviati a Santos. Oltre a ciò, lo sciopero danneggiò il rifornimento delle città, la posta, il sistema bancario e il trasporto delle persone” (p. 131). Questo sciopero provocava anche in Brasile strategie opposte: all'abbandono del posto di lavoro si reagiva con i crumiri; per giustificare l'intervento massiccio della polizia e dell'esercito si adducevano i danni alla proprietà delle compagnie ferroviarie; alla rivendicazione della dignità operaia si contrapponeva l'esigenza di mantenere l'ordine pubblico. Insomma, il solito contrappunto che accompagna uno sciopero riuscito. Però, in assenza di una precisa ricostruzione dei fatti, le ragioni degli scioperanti e quelle dei proprietari delle ferrovie (e quindi degli uffici pubblici che li appoggiavano) si presentano come due retoriche parallele e vuote di contenuto.

Siqueira sottolinea alcune aspetti giuridici dello sciopero: "In Brasile lo sciopero delle compagnie ferroviarie non era considerato uno sciopero dei servizi pubblici” (p. 123) e inoltre gli scioperanti chiedevano "l’applicazione di diritti positivi” e "garanzie statali”. Afferma che "qui è possibile fare un'antropofagia giuridica degli scioperi, non riducendoli a movimenti operai anarchici” (p. 122): l’antropofagia consisterebbe nel "percepire differenze tra gli scioperi brasiliani e quelli europei”, cioè - se intendo correttamente - nell'interpretare quell'evento in termini propriamente brasiliani, e non secondo schemi europei.

A mio giudizio, questa differenza non risulta con sufficiente chiarezza nel testo di Siqueira, che adduce sinteticamente, con riferimento all'inizio del secolo XX, la percezione in Francia degli scioperi ferroviari come l'interruzione d'un servizio pubblico e la forte limitazione del diritto di sciopero in Spagna. Il movimento brasiliano rivendicava invece un diritto statuito, quindi non si opponeva alle prescrizioni giuridiche e, per questo, non poteva essere definitivo anarchico.

c) L'applicazione del metodo “antropofagico" allo sciopero ferroviario del 1906. Ricostruiti così a grandi linee le norme e i fatti relativi allo sciopero del 1906, restano da valutare due risultati proposti dalla ricerca: in primo luogo, se ci sia stata una creazione di norme da parte degli scioperanti (quest'opera di Siqueira si presenta infatti come un capitolo della "storia del diritto prodotto dai movimenti sociali”); e, in secondo luogo, se e in quale misura "l'antropofagia giuridica” (evocata, più che elaborata, come metodo di questa ricerca) sia riconducibile ai concetti 
del modernismo brasiliano accennati nel $\S 3$, cioè se dello sciopero sia stata tracciata una storia specificamente brasiliana, e non una riproduzione delle storie giuridiche europee.

Sul primo punto, la risposta sembra venire dal testo stesso di Siqueira, che a più riprese constata che gli scioperanti rivendicavano un diritto esistente, del quale egli ha fornito i testi normativi di riferimento. A mio avviso, più che d'una creazione di diritto si tratta di differenti interpretazioni dello stesso testo normativo. Forse si potrebbe tentare di costruire una "storia del diritto prodotto dai movimenti sociali” individuando movimenti più creativi nelle loro rivendicazioni: ma anche in questo caso l'interpretazione a favore della creazione di diritto da parte dei movimenti sociali dipenderebbe dalla definizione di diritto su cui ci si fonda. Si ritornerebbe per questa via al dibattito tra concezioni formaliste e antiformaliste del diritto.

Il secondo punto - cioè a quali risultati conduca l'“antropofagia giuridica” - è difficile da valutare, perché la concezione antropofagica del modernismo brasiliano è di per sé sfuggente: nata come provocazione ricca di elementi ludici e ironici, tende ad esaurirsi nella protesta stessa, come si vede anche in molti testi del futurismo italiano; nata per essere applicata a prodotti artistici e letterari, si rivela a mio giudizio poco adatta ad essere applicata a testi normativi (e si ritorna per questa via ai dibattiti su diritto e letteratura); infine, la costruzione di una storia giuridica specificamente brasiliana sembra dipendere più dall’oggetto - come lo sciopero del 1906 fin qui esaminato - piuttosto che dal metodo. In altre parole, l'analisi di Siqueira è una proposta che va saggiata in più direzioni per verificare quali frutti possa dare.

Complessivamente l’opera di Siqueira è ricca di stimoli da approfondire e discutere. Se, da un lato, il timore di cadere nella vecchia storia della legislazione lo ha indotto a limitare troppo l'esposizione dei fatti legati allo sciopero, dall'altro l'enunciazione dell'evoluzione normativa sul diritto di sciopero in Brasile è un vero ausilio alla comprensione degli eventi (supra, lett. $a$ ).

Di rilevante interesse è anche la documentazione extragiuridica raccolta da Siqueira. Le prese di posizione pro e contro lo sciopero, i resoconti giornalistici, le minacce dirette delle imprese $^{32}$, i messaggi falsamente paternalistici ${ }^{33}$ e i volantini delle leghe operaie ampliano i confini della storia strettamente giuridica e chiariscono l'origine e gli effetti delle norme esaminate.

Si può quindi concordare con Gustavo Siqueira quando sintetizza così i risultati raggiunti dalla sua ricerca: "È interessante percepire con quanti nuovi elementi la 'storia del diritto prodotto dai movimenti sociali’ possa contribuire alla comprensione e alla discussione della storia del diritto

\footnotetext{
32 "Ogni operaio che domani non si presenti al lavoro sarà immediatamente licenziato" (p. 184, foto 3).

33 Dal manifestino intitolato "Un vecchio padre ai suoi figli spirituali in sciopero": "La Direzione della Companhia Paulista, credetemi, sta cooperando per il vostro bene"; "gli scioperi pregiudicano tutti, e soprattutto voi che dovete mantenere una famiglia”, e così via (p. 186, foto 5).
} 
d'un certo periodo: una ricerca sul diritto di sciopero del 1906 che ricorresse soltanto alla dottrina, alla legge o alle sentenze del giudiziario (specialmente se dei Tribunais Superiores) non rivelerebbe le diverse tensioni verificatesi giorno dopo giorno nel diritto di sciopero. Il diritto di sciopero consacrato dalle decisioni del potere giudiziario e dalla dottrina non poteva essere esercitato liberamente perché in pratica una parte sostanziale dello Stato non lo riconosceva” (p. 154). In conclusione, "la 'storia del diritto prodotto dai movimenti sociali' aiuta a percepire il diritto esercitato quotidianamente dalle persone, un diritto più vicino alla vita, alla quotidianità delle persone, un diritto vissuto dentro, ma anche oltre i tribunali” (p. 154).

Si deve condividere il giudizio positivo sull'ampliamento del campo di ricerca attraverso la documentazione sindacale, politica e comunque extra-giuridica delle parti in causa. Inoltre dobbiamo essere grati a Siqueira tanto per le notizie che ci apporta quanto per la nuova proposta metodologica, la quale pone una serie di quesiti: questi risultati in che relazione stanno con le premesse metodologiche, in particolare con il richiamo all'antropofagia giuridica che figura nel titolo? Indubbiamente la ricostruzione storico-giuridica dello sciopero ferroviario del 1906 porta la ricerca dentro al Brasile, la arricchisce di documenti sociali radicati in quella società (e in ciò ritorna alle esigenze espresse provocatoriamente della Settimana d’Arte Moderna di São Paulo). Ma è veramente una nuova metodologia? In che rapporto sta con la sociologia giuridica, con la sociologia storica del diritto, con la storia contemporanea del diritto?

Ognuno dei lettori di queste pagine darà una risposta fondata sulle proprie definizioni dei concetti-base fin qui evocati. Quindi gli interrogativi suscitati dalla ricerca di Siqueira riceveranno non una risposta, ma tante risposte. La discussione è aperta.

\section{FONTE E RIFERIMENTI}

ANDRADE, Oswald de. Memorie sentimentali di Giovanni Miramare. Tradução Giovanni Cutolo. Prefácio Giuseppe Ungaretti. Milano: Feltrinelli, 1970.

BOURDIEU, Pierre. Langage et pouvoir symbolique. Paris: Seuil, 2001.

O poder simbólico. Rio de Janeiro: Bertrand Brasil, 1989.

BROSZAT, Martin. Da Weimar a Hitler. Roma: Laterza, 2001.

CAPOGRASSI, Giuseppe. Il problema della scienza del diritto. Roma: Editrice del Foro Italiano, 1937.

. Opere. Milano: Giuffrè, 1959. v. 2. 
Carlos V. Recopilación 76. 23 de julio de 1523.

CARVALHO, Amilton Bueno de; Carvalho, Salo de (Org.). Direito alternativo brasileiro $e$ pensamento jurídico europeu. Rio de Janeiro: Lumen Juris, 2004.

FASSÒ, Guido. La storia come esperienza giuridica. Milano: Giuffrè, 1953.

FAUSTO, Boris. Trabalho urbano e conflito social: 1890-1920. São Paulo: Difel, 1977.

FONSECA, Maria Augusta. Oswald de Andrade (1890-1954): biografia. São Paulo (Secretaria de Estado da Cultura): Art Editora, 1990.

FONSECA, Ricardo Marcelo. O deserto e o vulcão. Reflexões e avaliações sobre a História do Direito no Brasil. Forum Historiae Juris, 15 jun. 2012. Disponível em: <http://www.forhistiur.de/zitat/1206fonseca.html>. Acesso em: 31 jan. 2015.

GOHN, Maria da Glória. Novas teorias dos movimentos sociais. São Paulo: Loyola, 2009.

- Teoria dos movimentos sociais: paradigmas clássicos e contemporâneos. São Paulo: Loyola, 1997.

GROSSI, Paolo. O direito entre poder e ordenamento. Belo Horizonte: Del Rey, 2010.

HÄNEL, Gustav. Über Gültigkeit des römischen Rechts in Portugal bis zu dem Jahre 1791. Kritische Zeitschrift für Rechtswissenschaft und Gesetzgebung des Auslandes, 1835.

HESPANHA, António M. A história do direito na história social. Lisboa: Livros Horizonte, 1978. . Cultura jurídica europeia. Síntese de um milênio. Florianópolis: Fundação Boiteux, 2005.

O caleidoscópio do direito. O direito e a justiça nos dias e no mundo de hoje. Coimbra: Almedina, 2007.

HOBSBAWM, Eric. Rebeldes primitivos. Estudio sobre las formas arcaicas de los movimientos sociales en los siglos XIX y XX. Barcelona: Crítica, 2001.

HUNGRIA, Nelson. Compêndio de direito penal. Rio de Janeiro: Jacyntho, 1936.

ISER, Wolfgang. Der Akt des Lesens. München: Fink, 1976.

JÁUREGUI, Carlos A. Canibalia: canibalismo, calibanismo y antropología cultural en América Latina. Madrid: Iberoamericana, 2008.

JAUSS, Hans Robert. A literatura como provocação. História da literatura como provocação literária. Lisboa: Vega, 1993.

Literaturgeschichte als Provokation der Literaturwissenschaft. Konstanz: Konstanzer Universitätsreden, 1967. 
. Literaturgeschichte als Provokation. Frankfurt am Main: Suhrkamp, 1970.

KOSELLEK, Reinhart. Futuro passado. Contribuição à semântica dos tempos históricos. Rio de Janeiro: Contraponto, PUC-RJ, 2006.

LEBEK, Wolfgang Dieter. Kannibalen und Kariben auf der Ersten Reise des Kolumbus. In: FULDA, Daniel; PAPE, Walter (Ed.). Das andere Essen. Kannibalismus als Motiv und Metapher in der Literatur. Freiburg im Breisgau: Rombach Verlag, 2001, p. 53-112.

LOSANO, Mario G. Función Social de la Propiedad y los Latifundios Ocupados. Los Sin Tierra de Brasil. Madrid: Dykinson, 2006.

. Il Movimento Sem Terra del Brasile. Funzione sociale della proprietà e latifondi occupati. Reggio Emilia: Diabasis, 2007.

. La legge e la zappa: origini e sviluppi del diritto alternativo in Europa e in Sudamerica. Materiali per una storia della cultura giuridica, ano 30, n. 1, Bologna, Il Mulino, 2000. Tradução para o espanhol, por Andrea Greppi, disponível em: <http:/earchivo.uc3m.es/bitstream/10016/1384/1/DyL-2000-V-8-Losano.pdf>. Acesso em: 31 jan. 2015.

. Storia contemporanea del diritto e sociologia storica. Milano: Franco Angeli, 1997.

2000.

. Un giurista tropicale. Tobias Barreto fra Brasile reale e Germania ideale. Roma: Laterza,

MARTINS JR., José Isidoro. História do direito nacional. Rio de Janeiro: Typographia da Empreza Democratica Editora, 1895.

MORAES, Evaristo de. Apontamentos de direito operário. Ed. fac-sim. São Paulo: Ltr, 1998.

OPOCHER, Enrico. Lezioni di filosofia del diritto. Padova: Cedam, 1983.

PICCHIO, Luciana Stegagno. Storia della letteratura brasiliana. Firenze: Sansoni, 1972.

REALE, Miguel. O direito como experiência. Introdução à epistemologia jurídica. São Paulo: Saraiva, 1968.

SANTOS, Boaventura de Sousa. O discurso e o poder. Ensaio sobre a sociologia da retórica jurídica. Porto Alegre: Fabris, 1988.

SCHERER-WARREN, Ilse. Redes de movimentos sociais. São Paulo: Loyola, 1993.

SFORZA, Widar Cesarini. Filosofia del diritto. Milano: Giuffrè, 1958.

SILVA, Joseane Lucia. "L'anthropophagisme" dans l'identité culturelle brésilienne. Paris: Harmattan, 2009.

SIQUEIRA, Galdino. Direito penal brasileiro. 2. ed. Rio de Janeiro: Jacyntho, 1932, 2 v. 
SIQUEIRA, Gustavo Silveira. História do direito pelos movimentos sociais. Cidadania, Experiências e Antropofagia jurídica nas Estradas de Ferro (Brasil, 1906). Rio de Janeiro: Lumen Juris - Faperj, 2014.

. Pensamiento y norma. La contribución del concepto de experiencia jurídica para la historia del derecho. In: FERRARI, Anderson et al. Horizontes de Brasil. Barcelona: APEC, 2011.

SLATOFF, Walter J. With Respect to Readers. Dimensions of Literary Response. Ithaca, NY: Cornell University Press, 1970.

TRINDADE, Hélgio. Integralismo: o fascismo brasileiro na década de 30. São Paulo: Difel, 1979.

UHSE, Bodo. Tagebuch aus China. Berlin, DDR: Aufbau-Verlag, 1956.

WALDMAN, Thaís. À “frente” da Semana de Arte Moderna: a presença de Graça Aranha e Paulo Prado. Estudos históricos, Rio de Janeiro, vol. 23, jan./jun. 2010, n. 45. Disponível em: $<$ http://www.scielo.br/scielo.php?pid=S0103-21862010000100004\&script=sci_arttext $>$. Acesso em: 31 jan. 2015.

\section{LEGAL AND HISTORICAL SOCIOLOGY, LEGAL HISTORY, AND, IN BRAZIL, “JURIDICAL ANTHROPOPHAGY”}

ABSTRACT: Through the analysis of the book História do direito pelos movimentos sociais, by Gustavo Silveira Siqueira, this article studies, in the prism of legal theory, the problems and possibilities of the so-called "juridical anthropophagy", the methodological approach proposed by Siqueira for Brazilian legal history.

KEYWORDS: Brazilian legal history. “Juridical Anthropophagy”. Legal theory. Methodology. 